\title{
Now Hear This: Auditory Sense may be an Undervalued Component of Effective Modeling and Imagery Interventions in Sport
}

\author{
Jenny $\mathrm{O}^{1, *}$, Barbi Law ${ }^{2}$ and Amanda Rymal ${ }^{3}$ \\ ${ }^{I}$ Department of Kinesiology, California State University East Bay, Hayward, USA; ${ }^{2}$ School of Physical and Health \\ Education, Nipissing University, North Bay, Canada; ${ }^{3}$ Department of Kinesiology, California State University San \\ Bernardino, San Bernardino, USA
}

\begin{abstract}
One of the most important goals of behavioral research in sport psychology and motor learning is to increase our understanding of how to more effectively manipulate structural elements of psychological skills so as to optimize learning and performance. Imagery and modeling research have long-held parallel trajectories; advancements in the understanding of one construct has often informed subsequent research on the other. Preliminary research examining the effect of auditory modeling has indicated that deliberate manipulation of sounds employed during modeled actions can positively impact motor skill learning, performance, and consistency. The imagery research has yet to directly examine the auditory sense, and thus examination of this imagery component would represent a meaningful contribution to our understanding of how to further optimize athletes' imagery practice. The current paper reviews current knowledge regarding effective imagery and modeling structure, and provides theoretical and evidence-based rationales for the examination of the auditory sense in imagery research.
\end{abstract}

Keywords: Auditory sense, bio-informational theory, imagery, intervention, modeling, psychological skills, sport.

\section{INTRODUCTION}

The primary goal for a developing competitive athlete is to improve his or her subjective skill level. However, as he or she becomes more skilled and progresses to more challenging levels of competition, performance consistency is just as important as skill development. Inconsistency in performance can have detrimental effects in sport. These effects can range in severity: one may commit isolated performance errors, lose a competition, experience acute or chronic negative psychological consequences (e.g., decreases in confidence and/or motivation and/or increases in anxiety), or perhaps, even experience injury.

To assist competitive athletes with both skill development and performance consistency, psychological skills are often adopted. Two such skills are imagery and modeling. Modeling is also referred to in the sport psychology literature as observational learning; however, we believe that given the context of the current paper - a focus on the auditory sense, modeling is the more appropriate term, here. Research on imagery and modeling have long-held parallel trajectories; advancements in the understanding of one construct has often informed subsequent research on the other [1-3]. In the recent years, the examination of auditory modeling has revealed the auditory sense to be an important factor in motor skill learning, performance, and consistency [4-7]. Given the dearth of imagery research focused on the auditory sense, examination of this imagery component would

*Address correspondence to this author at the Department of Kinesiology, California State University East Bay, Hayward, USA;

Tel: 510-885-2907; Fax: 510-885-2423; E-mail: jenny.o@csueastbay.edu represent a meaningful contribution to our understanding of how to optimize athletes' imagery practice. The deliberate use of sport-specific sounds may be an undervalued component of effective images.

\section{IMAGERY IN SPORT}

Mental imagery is one of the most researched and widely-implemented psychological skills in sport [8]. In fact, Silva and Stevens [9] have suggested that imagery is often viewed as "the cornerstone of sport psychology interventions" (p. 206) as it is frequently employed by athletes to aid in the development, mastery, and performance of sport skills and strategies, as well as in the experience of psychological outcomes. Morris, Spittle, and Watt [10] have advanced a working definition of sport imagery that captures the essential elements of this psychological skill:

Imagery, in the context of sport, may be considered as the creation or re-creation of an experience generated from memorial information, involving quasi-sensorial, quasiperceptual, and quasi-affective characteristics, that is under volitional control of the imager, and which may occur in the absence of the real stimulus antecedents normally associated with the actual experience (p. 19).

The examination of imagery use in sport has pursued a variety of research topics. A large body of research has examined the effectiveness of imagery in facilitating sport skill and strategy learning and performance [11-13] as well as positive psychological outcomes such as reductions in anxiety [14] and increases in motivation [15], self-efficacy [16], and confidence [17]. The facilitative effect of imagery on sport performance is well-documented in the research literature. 
Several theories have been proposed to explain how imagery exerts influence on sport performance and experience [18]. One such theory is Lang's [19, 20] Bio-informational theory. Lang posited that images are comprised of stimulus, response, and meaning propositions. Stimulus propositions represent the salient features of an image, such as particular human senses and/or emotions, used to construct and experience the image. Response propositions involve the various cognitive, behavioral, and physiological responses one has to the stimuli in the image. Meaning propositions are a highly subjective component of the imagery experience, representing the individual's interpretation of the image, relative to the hypothetical consequences of what was imaged. Based on the tenets of the bio-informational theory, effective imagery requires an individual achieve high subjective meaning of the image, through deliberate manipulation of stimulus and response propositions.

One body of sport imagery research which has increased our understanding of how one might more effectively manipulate stimulus and response propositions concerns examinations and subsequent explanations of the relevance of imagery use to sport performance [2,21]. Paivio [21] was the first to propose an analytic framework that explained how imagery influences sport and physical activity. A number of years later, Hall and his colleagues [2] expanded upon Paivio's conceptual model of the functions of imagery use. It is now well-established that sport imagery can serve a cognitive and/or a motivational function, and that each function operates on either a specific or general level. The five functions of sport imagery are: cognitive general (CG; i.e., images intended to assist in the learning, development, or mastery of strategies, game plans, or routines); cognitive specific (CS; i.e., images used to assist in the learning, development, or mastery of specific sport skills); motivation specific (MS; images which assist with increasing intensity of effort during practice or competition); motivation general-arousal (MG-A; images intended to regulate arousal and anxiety); and, motivation general-mastery (MG-M; images employed to increase mental toughness, perceptions of control, selfconfidence, and self-efficacy). These five functions of imagery represent the various reasons why athletes choose to employ imagery in sport. Moreover, deliberate selection of the imagery function(s) of one's image would certainly influence the stimulus and response propositions of that image.

For example, a golfer wanting to employ imagery to increase her self-efficacy for an upcoming bunker shot (i.e., MG-M imagery) may choose to use sight, kinaesthetic and tactile feel, and sound (stimulus propositions) to generate an image wherein she focuses on feeling optimally focused and in total control when hitting the shot (cognitive response propositions). Based on Langian [19, 20] conceptions, such deliberate image structure and experience should result in enhanced meaning propositions (i.e., "I can make this shot"; enhanced self-efficacy for the bunker shot). Research has demonstrated that selecting the appropriate function of imagery is important in achieving the intended outcome of the imagery session [22, 23]. Although this imagery focusimagery function match may be paramount to effective imagery use, researchers suggest that other factors also need to be considered to optimize imagery's effectiveness [24, 25].
One such factor is imagery content; the structural elements of an athlete's images. By definition, imagery content is a key element in defining the stimulus propositions of one's image. Research has identified several factors that comprise imagery content: surroundings (e.g., environment); nature (i.e., positive vs. negative outcomes); effectiveness (i.e., helpful or hurtful images); perspective (i.e., internal vs. external); speed (i.e., slow motion vs. real-time vs. fast motion); sessions (i.e., duration and frequency); and modalities (i.e., use of selected human senses) [26-28].

Regarding imagery modalities, research by Kosslyn, Seger, Pani, and Hillger [29] indicated that the visual sense was the predominant sense reported during imagery. Certainly, this tendency to emphasize the visual sense in images (over other senses) is reflected in the popular use of the term "visualization" as a synonym for imagery practice. The term visualization, however, is problematic; it suggests that 'seeing' one's images is the only relevant stimulus proposition concern for imagers despite the fact that imagery literature has clearly indicated that the most effective images are selectively multimodal in nature (with respect to the senses) $[18,24,25]$.

\section{MODELING IN SPORT}

Modeling refers to the process of viewing a demonstration and then modifying our own behavior based on that experience [30]. In fact, Bandura suggests that the majority of human behavior is acquired through modeling. Within the motor learning domain, it has been well established that modeling is effective for facilitating motor skill acquisition and performance [31-33]. Modeling has also gained popularity in applied sport psychology with highlight music videotapes being employed in an attempt to enhance athlete performance and psychological outcomes [34-36]. In addition to the physical performance benefits of modeling, research supports its validity for creating positive psychological changes such as enhanced self-efficacy [37-41], selfsatisfaction [38], and motivation [42], as well as reduced anxiety $[41,43]$.

The dominant explanation used as the foundation for most of the modeling research to date has been Bandura's [30] social cognitive theory, as it accounts for both physical and psychological performance improvements. In order for an action or belief to be modified through modeling, the observer must pay attention to relevant features of a demonstration and must retain that information in his or her memory. The action or belief must be valued, such that the observer is motivated to produce the behavior when an opportunity arises. According to social cognitive theory, the greater the perceived model-observer similarity, the more effective the demonstration will be in modifying the observer's cognitions and behaviors. Bandura [30] recognizes that the individual's beliefs interact with environmental features to produce behavioral responses.

We also propose that Lang's bio-informational theory $[19,20]$ can be applied to help understand the effects of modeling on learning and performance. In carefully and deliberately structuring the various elements of a modeled action, high relevance and individualization of stimulus propositions of the model will be achieved. Consequently, re- 
sponse propositions within the modeled action will more accurately reflect the current performance and/or performance goals of the learner. This accuracy in stimulus and response propositions should subsequently influence meaning propositions; if the modeled action (i.e., stimulus propositions) is perceived as highly relevant to the learner and the behavioral responses (i.e., response propositions) highly desirable, the learner is likely to perceive that the modeled action is achievable and that he or she wants to achieve them (i.e., meaning propositions reflecting increased self-efficacy and greater self-determined motivation for skill learning and performance).

Much of the recent research exploring modeling within sport psychology has focused on understanding how it is used by the athletes themselves. Based on the notion that imagery and modeling share similar cognitive processes [30, 31, 44, 45], Cumming and colleagues [1] applied Paivio's [21] analytic framework for imagery effects to the study of modeling. They established that modeling appears to serve three functions in sport. The skill function refers to modeling used to develop and execute sport skills, similar to CS imagery. The strategy function refers to modeling used to develop and execute strategies, game plans, and routines, similar to CG imagery. The performance function refers to modeling used to create optimal arousal levels and mental states for performance, similar to MG-M and MG-A imagery combined. Use of a particular function of modeling results in a corresponding change in a related psychological variable. For example, Law and Hall [46] found that among adults learning an independent sport (e.g., golf), use of the skill function, but not use of the strategy or performance functions, was a significant predictor of their self-efficacy to acquire the skills necessary to play the sport. While not explicitly designed to target the performance function, research employing a coping model (i.e., a model who improves in skill level and who expresses increasingly positive selfbeliefs and ability to cope with a challenge) demonstrated improvements in self-efficacy as well as performance $[37,47]$. Modeling interventions designed to target specific functions is a key area in need of future research.

It should be noted that effective modeling use relies not only on a match between the function used and the intended outcome. Through a comprehensive review of the modeling literature, Ste-Marie, Law, Rymal, O, Hall, \& McCullagh [48] developed an Applied Model for the Use of Observation (AMUO) that highlights key factors to consider when designing effective modeling experiences. One such factor is the specific characteristics of the demonstration (a concept parallel to imagery content in the sport imagery literature). Research has supported the notion that characteristics of the demonstration are important for creating effective modeling interventions. These include: model type (skilled, learning, mastery, coping, self), modality (live, video), instructional features (verbal cues, rehearsal strategies), speed (slow motion, real time), viewing angle (face-to-face, side, behind), timing (before, during, after skill performance), frequency of viewings provided, and the degree of self-control (i.e., choice) the learner has over when to view the demonstration $[33,48]$. For example, a coach attempting to build confidence in a young athlete who is playing soccer for the first time may point out a teammate who was new to the team last year and who has shown great improvement. Or, the coach may put together a highlight tape of the young athlete showing in real-time how her skills have improved from the beginning to the end of the season. The coach may suggest that the athlete watches this video when she is feeling less confident or as part of her pre-game preparation.

Based on our review of the current understanding of sport imagery and modeling, it is clear that effective imagery is much more complex than "seeing is believing", and effective modeling, much more complex than "watch and learn."

\section{LITERATURE REVIEW}

The literature on sport imagery and modeling has evolved in the past several decades from preliminary investigations intended to describe these phenomena to research attempting to identify ways in which sport imagery and modeling may be optimized. For example, following several anecdotal reports of differential image speed use by athletes $[49,27], \mathrm{O}$ and Munroe-Chandler [50] examined the effect of three CS image speed conditions on the performance of a soccer dribbling task (i.e., dribbling around a set of cones). Ninety-seven participants were randomly assigned to one of five conditions: real-time imagery, slow motion imagery, slow motion concluded with real-time imagery, physical practice, or control. Participants were instructed that the goal of the task was to dribble around the set of cones as quickly and as accurately as possible (i.e., avoiding contact with cones while maintaining control of the soccer ball). During the intervention period, participants were explicitly instructed to image at their designated speed and were provided with a functional goal of their image speed use (e.g., "use slow motion [real-time] speed of your image to help you learn how to dribble the soccer ball faster and more accurately"). Results indicated all three image speed conditions and the physical practice condition outperformed the control condition, and significantly improved time and error performance to the same degree following the intervention phase. These findings conflicted with the existing image speed guideline which emphasized the importance of ensuring image speed match real-time physical execution speed [51]. As a result of this and subsequent research examinations and theoretical discussions of differential image speed use $[28,52]$, current image speed guidelines now advocate deliberate selection of image speed to enhance imagery's effectiveness [53, 54].

Similar investigations have increased our understanding of how to optimize athletes' modeling use. Modeling intervention studies often employ skilled or expert models [48]; consistent with a common assumption that an expert model may be most effective [55]. However, research has shown that observing either a correct model or a learning model (i.e., one who gradually improves in performance) can be effective for enhancing skill acquisition [56-58]. Observing a peer coping model (i.e., a fellow learner who shows gradual skill improvement and progressively more positive cognitions) can be beneficial for increasing self-efficacy beliefs, while observing a peer mastery model (i.e., a fellow learner who always shows correct performance and positive cognitions) can be beneficial for improving skill performance $[37,41]$. Such findings have been incorporated into contem- 
porary applied recommendations for effective modeling use $[33,48]$.

Recently, some modeling research has focused on the role of sound in enhancing motor learning and performance. Preliminary data have indicated that auditory modeling can improve movement perception and reproduction [4, 5, 7], relative timing $[6,59,60]$, and absolute performance [61]. From an applied perspective, the ultimate goal of cognitivebehavioral research is to enable evidence-based recommendations for practitioners. Moreover, these recommendations should be considered amenable to change; as new research is conducted and findings adequately replicated, recommendations should be revised and updated to reflect our current understanding of how to most effectively manipulate motor learning conditions.

Accordingly, some of the auditory modeling research provides preliminary support for the use of "second order biofeedback techniques" (SOBF) [61]. This type of auditory modeling uses natural movement sounds as a learning tool (e.g., the recorded sound of a golf club moving through the air during a swing, the recorded sound of tennis ball-tennis racquet contact during a serve or hit, etc.). Furthermore, more comprehensive review and synthesis of the modeling literature seems to provide evidence to support the hypothesis that SOBF of one's own movements (i.e., "self-model") $[62,63]$ may be the most efficacious way in which to use auditory modeling to facilitate skill learning and performance $[38,61,64]$. Given the limited research on the effects of self-model SOBF on skill learning and performance [61], we strongly recommend further and more direct research examinations of self-model SOBF prior to the advancement of practical recommendations.

We also see opportunities for imagery researchers. Given the identified similarities in brain activation while imaging and engaging in modeling [65-68], the noted parallelism between evidence-based imagery and modeling recommendations in sport [25, 48], and the lack of direct research on the effects of imagery interventions employing the auditory sense, imagery research examining the influence of the auditory sense on sport skill learning and performance is certainly warranted. The remainder of this paper presents our rationale and supporting evidence for our call for further research on the effects of self-model SOBF and auditory imagery on sport skill learning and performance.

\section{AUDITORY MODELING IS EFFECTIVE}

Cesari, Camponogara, Papetti, Roccesso, and Fontana [69] examined whether skill level moderated the effects of hearing movement-relevant sounds on action anticipation and performance of a simulated skateboard-jump task. Participants $(N=20)$ were either skilled or unskilled skateboarders. Researchers used movement sonification (i.e., synthesized sounds that represents kinematic and/or dynamic movement parameters of a skill) to model the sounds that a skateboard would make while executing a jump. Participants were not provided any corresponding visual modeling of the jump. Results indicated that skill level did in fact moderate the effect of auditory modeling on action anticipation and performance. Compared to the unskilled participants, skaters (skilled participants) more accurately used skateboard sounds to anticipate the upcoming skateboard jump and more accurately made postural adjustments and body movements consistent with those which would occur when physically executing the jump. This may suggest that more skilled performers have stronger mental representations of sportspecific skills, that these representations are comprised of information that can be accessed using more than just visual stimuli, and that more skilled performers can use these representations to make more accurate behavioral decisions. Accordingly, this might also indicate that it is important for movement practitioners to focus on developing multi-modal mental representations for sport-specific skills in more novice performers to facilitate greater skill learning and performance.

Although movement sonification appears to be an effective method with which to facilitate certain aspects of skill performance, sonifying movement sounds may not be the most practical method in real-world sport skill learning situations (e.g., coaches, physical education teachers, and other movement practitioners may not have the proper training, nor access to the specialized equipment required to accurately sonify movement). However, this does not suggest that the use of movement sounds to facilitate learning be relegated exclusively to highly-controlled laboratory situations. Of particular relevance to the current discussion is the relatively recent auditory modeling research examining SOBF [61-71]. Such research has highlighted potentially facilitative effects of SOBF on motor learning and performance. Agostini and colleagues [61] examined the effect of the provision of SOBF on skill performance (i.e., distance thrown) and consistency (i.e., inter-throw variability) of a hammer throw. Five elite hammer throwers performed 10 baseline throws, followed by a five-minute rest, and then the experimental condition. The experimental condition consisted of the throwers being primed with five SOBF trials of their best throw from the baseline phase before physically performing each of 10 throws. Results indicated that average throw distance significantly increased and throw variability significantly decreased (i.e., absolute performance improved) in the experimental condition. Despite the small sample size and the lack of a true control group (to control for the possibility of physical practice effects), these results represent promising preliminary evidence of the facilitative effects of using natural sounds to prime movement. A limitation of the current examination, however, is that the authors failed to directly assess possible mechanisms underlying the increases in absolute performance. For example, it is unknown as to whether performance changes were due to changes in technique, throwing strategy, and/or perhaps changes in psychological factors such as motivation, self-efficacy, or focus of attention. Inclusion of analyses designed to identify potential mechanisms of change would have provided meaningful information for movement practitioners (to inform instruction and feedback) as well as future SOBF researchers.

More recent research has corroborated the findings of Agostini and colleagues [61] regarding the efficacy of SOBF in eliciting positive changes in motor performance outcomes. For example, Murgia and colleagues [71] created a breath regulation intervention using a within-subjects design. Participants were asked to synchronize their inhalations relative to a SOBF presentation of their breathing, a sonified presen- 
tation of their breathing, and, were asked to simply breathe normally (conditions were counter-balanced among participants). Results indicated that only the SOBF condition elicited significant decreases in breath variability, thus providing evidence that SOBF can contribute unique influence on performance (breath variability) compared to sonified breath sounds and control conditions.

From a bio-informational theory perspective $[19,20]$ the deliberate use of natural movement sounds within modeled actions (SOBF) seems logical. SOBF can be viewed as a direct manipulation of stimulus propositions. Theoretically, increasing the vividness and representativeness of the sounds heard during a modeled action should increase the effect of the modeled information (i.e., stimulus propositions) on subsequent response and meaning propositions. For example, a PE teacher may record the sounds made during the approach and ball strike phases of a soccer penalty kick. These natural movement sounds may be played to a PE student about to perform a penalty kick practice trial and the student cued to use the SOBF to obtain important auditory information regarding the relative timing of proper penalty kick execution. Subsequently, the student would then perform the practice trial, and to focus on making deliberate attempts to replicate the timing of the movement sounds heard.

In addition to preliminary evidence of positive effects on absolute performance $[61,71,72]$, the auditory modeling literature also supports the effectiveness of movement sounds in facilitating learning of the relative timing of motor skills [5, 59, 60]. Lai and colleagues [59] used a keyboard button-pressing task and asked participants to learn a prescribed timing sequence (i.e., the key sequence had to be performed with a particular rhythm, thus representing a task which emphasized the learning of relative timing). Learning and retention during three practice conditions were compared: 1) $100 \%$ auditory modeling (sounds representing the relative timing of the task presented during all practice trials), 2) 50\% auditory modeling, and, 3) $0 \%$ auditory modeling. Results indicated that the $100 \%$ and $50 \%$ auditory modeling practice conditions had better learning and retention trial performance compared to the $0 \%$ auditory modeling condition, thus suggesting that the provision of movement sounds during practice can provide highly relevant information to learners regarding relative timing of a movement. From a practical perspective, these findings highlight the importance of finding ways to effectively and efficiently teach the relative timing of motor skills, as frequently, time is a limited resource for movement practitioners (e.g., PE teachers, physical therapists, coaches, etc.).

\section{USING SELF-MODELS MAY OPTIMIZE SOBF}

In a recent examination, Murgia et al. [64] found that expert golfers were able to discriminate their own golf swings from those of others based only on auditory information (i.e., the sound of different golf swings). This discriminatory behavior held even when other aurally-presented swings had similar temporal structures. Similar results were found by Kennel and colleagues [70]; participants were able to distinguish between self- and other-produced movements of hurdling performance. This finding suggests that individuals may code and store their own unique movements not just in terms of visual and kinaesthetic properties, but also in terms of auditory properties. Consequently, it is reasonable to assert that individuals have highly individualized mental representations for learned movements.

Assuming these highly-individualized mental representations, it is reasonable to hypothesize that the most effective auditory model to use with learners working toward skill mastery would be a self-model. Self-modeling uses individuals' best performances as learning stimuli [72]. This hypothesis has both theoretical and evidence-based support. Theoretically [19, 20], using sounds of one's own movements would increase the accuracy and meaningfulness of the stimulus propositions employed during learning trials as the auditory stimuli would be reflective of that created by the learner's unique movements during subjective best performances. This would lead to more accurate response propositions (i.e., behavioral responses more reflective of the performer's actual movements during subjective best performances), and subsequently, would lead to more efficient changes in motor performance as skill level develops (assuming that self-model SOBF is updated as skill level improves - although this assertion has yet to be empirically tested). Meaning propositions may also be positively affected, possibly reflected in increased perceived competence and control of one's movements.

The self-modeling research also supports the use of one's own movement sounds during SOBF modeling. A substantial body of the modeling literature has examined the differential effects of employing different model types [37, 41, 72$75,86]$. Results have consistently indicated that the more similar the learner perceives the model to be, the more effective is subsequent learning. Thus, in employing a self-model, one is essentially maximizing model similarity, and therefore, potentially maximizing learning effects of the modeling intervention.

\section{SIMILARITIES BETWEEN MODELING AND IM- AGERY}

Evidence of the similarities between modeling and imagery can be found in both the neuropsychology and behavioral sport psychology literature. From a neuropsychological perspective, di Pellegrino, Fadiga, Fogassi, Gallese, \& Rizzolatti [76] first observed co-activation in the areas of the brain associated with movement (i.e., F5 pre-motor cortex and posterior parietal area) in macaque monkeys who were merely observing the movements of other monkeys. Since this landmark study, similar findings have been observed in humans. For example, and more specific to the discussion in the current paper, research has supported the hypothesis that auditory imagery activates brain areas similar to that of auditory perception $[77,78]$. Moreover, it appears that areas of the brain that are activated during both imagery and modeling are also activated when an individual physically performs a skill [65, 66]. In fact, researchers have suggested that modeling and imagery may actually prime motor execution by initiating motor planning processes in the brain $[68,79]$.

From the behavioral sport psychology literature, there exists very clear parallelism between practical recommendations advanced for the structure of modeling and imagery 
experiences. These recommendations are based on extensive descriptive research examining athletes' preferential use of imagery and modeling, as well as experimental and quasiexperimental research examining the differential effects of various imagery and modeling conditions. This large body of research has been synthesized into two separate applied models intended to offer 'best-practices' for structuring imagery and modeling interventions as well as to guide future research (i.e., Applied Model for Imagery Use; AMIU [25]; AMUO [48]). Examination of these applied models reveals high similarity between factors identified as being important to effective intervention design. For example, both models present information regarding the 'who', 'what', 'why', 'when', and 'where' of intervention design. Regarding the 'who' of imagery and modeling, it is recommended that practitioners deliberately select the actor to be employed within the imagery or modeling practice, based on the personal characteristics of the person using the psychological skill (e.g., skill level, physical characteristics, etc.). Regardless of whom the actor is both the AMIU and the AMUO note that maximizing perceived similarity between actor and the individual using the image or model is paramount. 'What' recommendations for imagery and modeling also parallel each other with respect to suggestions concerning imagery and modeling perspective, timing, and speed, while similarities in the 'why' of modeling and imagery is evident in the identification of the various functions, respectively, of the two psychological skills. Regarding the 'when' and 'where' of modeling and imagery, recommendations generally suggest that these factors are largely a matter of personal preference and feasibility of use.

Collectively, based on the evidence supporting similar brain region activation during imagery and modeling as well as the similarities noted in recommended structure of use of these two psychological skills, it can be argued that imagery and modeling elicit very similar effects on motor skill learning and performance. How similar these effects actually are is currently unknown; the literature has noted that limited research has attempted to identify the differential effects of imagery and modeling [33] and that this certainly represents an area in great need of further research.

\section{THE AUDITORY SENSE IS UNDER-RESEARCHED}

Another area in which the sport imagery and modeling literature exhibit similarity concerns the (limited) understanding of the role of the auditory sense. Although the AMIU [25] recommends employing as many of the senses as are relevant to maximize the representativeness of one's imagery (i.e., how closely the imaged experience parallels 'real life' experience), the current authors were not able to locate any sport imagery research that has directly examined the role that sounds play in sport imagery's effectiveness. Similarly, the AMUO [48] actually fails to address the inclusion of natural movement sounds in modeled actions, however, this may be due to the AMUO's almost exclusive focus on the visual aspects of model structure (the AMUO does address the use of verbal cues). As reviewed earlier in this paper, some promising preliminary research does exist examining the effect of auditory modeling on motor learning and performance [4-7, 59-61], but this research certainly needs to be expanded upon before it can be confidently translated into practical recommendations.

Some sport imagery literature has noted that athletes do report making use of the auditory sense within their images $[27,53]$; however, this evidence is anecdotal in nature (i.e., based on athlete interviews). More research is needed to more systematically examine whether, how, and for what functions athletes are making use of natural movement sounds within their sport images. Although the effects of use of the auditory sense in images on sport skill learning and performance has yet to be directly examined in the sport domain, it has been extensively researched in language and music [80]. It is likely that review of this auditory imagery literature, and in particular, the literature examining the role of the auditory sense in learning and performing on musical instruments (given the kinaesthetic components involved in both sport actions and musical instrument performance), can inform future sport imagery research. In addition, further insights may also be drawn from the (limited) auditory modeling research given the noted cognitive processing and structural parallels between modeling and imagery.

Intons-Peterson [81] found that individuals instructed to image familiar sounds made by everyday objects almost always reported concurrent visual images; however, when instructed to generate a visual image, individuals reported concurrent auditory images approximately $50 \%$ of the time. This finding is substantiated by the findings of Kosslyn et al. [29], who found that the visual sense was the most reported sense employed by adults during imagery. Given the large emphasis placed on the visual and kinaesthetic aspects of sport imagery $[82,83]$, as well as the tendency to rely heavily on the visual sense during imagery experiences $[29,81]$, it is possible that athletes currently underutilize the auditory sense within their images.

For example, current sport imagery ability measures such as the Movement Imagery Questionnaire-Revised (MIQ-R) [82] and the Vividness of Movement Imagery Questionnaire2 (VMIQ-2) [83] only assess the quality of the visual and kinaesthetic components of movement imagery. Thus, sport imagery practitioners using these popular imagery ability assessment tools do not gain insight into whether, and how well athletes are incorporating sounds into their imagery experiences. Consequently, practitioners cannot efficiently train individuals to use the auditory sense within their images given the absence of an objective measure of current auditory imagery ability. Moreover, when providing evidence-based imagery training to athletes, imagery practitioners are more likely to emphasize the visual and kinaesthetic senses given the relatively exclusive focus of the sport imagery research on these two senses $[18,81,82]$.

Of course, concern about the quality of an athlete's use of the auditory sense during imagery is a moot point if sounds are not found to contribute meaningfully to skill learning and/or performance. Research from the music domain, however, indicates that incorporation of movement sounds within one's images may in fact include information facilitative of motor planning and execution [79, 84, 85]. For example, Keller and Appel [79] examined the coordination of body sway and sound onsets in seven piano duets during a task that required the duets to play several coordinated but con- 
trasting musical sequences. The degree of coordination between the duets (i.e., performance quality) was found to be positively correlated with auditory imagery ability (which was assessed by researchers prior to performing the piano duet task). Moreover, this significant correlation held regardless of whether the duet was in visual contact with each other during piano performance, thus suggesting that the coordination of body sway and sound onset during piano duets was not exclusively governed by visual cues. The researchers asserted that auditory imagery facilitated action anticipation (i.e., when to initiate keystrokes and body sway). With regard to sport imagery, it is possible that the inclusion of movement sounds can influence action anticipation (and consequently, learning and performance). For example, a figure skater using imagery to assist with learning a double axel (i.e., a technical jump in figure skating) may incorporate and heavily focus on the sound of his or her skate blades against the ice as he or she transitions through the various jump phases of the double axel (i.e., entry, take-off, air position, and landing). This sound is distinctly audible during actual physical performance (in the absence of ambient noise), and based on preliminary findings from the SOBF auditory modeling literature [61] and the noted similarities between modeling and imagery, may provide critical movement information regarding relative timing of the skill. Sport imagery literature has yet to systematically explore this potentially facilitative effect of natural movement sounds in imagery.

\section{CONCLUDING REMARKS AND RECOMMENDA- TIONS FOR FUTURE RESEARCH}

In summary, ample research evidence exists to support the cognitive processing similarities between imagery and modeling. Moreover, cognitive-behavioral imagery and modeling research have long-held parallel trajectories with respect to the identification of important structural features that need to be considered when designing imagery and modeling interventions. We feel that we have offered some interesting discussion in the current paper as well as providing theoretical and evidence-based support for potential applications of natural movement sounds within imagery and modeling. However, given the limited research on the use of auditory modeling in sport applications and the complete absence of imagery research directly examining the auditory sense, we strongly recommend that applied modeling and imagery researchers consider these two topics in future research endeavors.

Based on the current limitations in research examining the role of movement sounds in motor learning and performance, we offer several avenues for future research: 1) gaining a stronger understanding of the deliberate and spontaneous use of movement sounds (natural or simulated) by athletes when learning or performing sport-specific skills (i.e., description), 2) attempting to determine what unique contributions auditory information provides a learner/performer when learning and/or performing sport skills (i.e., explanation), and 3) performing more extensive and rigorous examinations of the (potentially differential) effects of movement sonification and SOBF on skill learning and performance (i.e., employing larger sample sizes and true control groups, employing multiple experimental groups and participant skill levels to enable within and between-group comparisons, and attempting to identify mechanisms of learning and performance change; prediction). We believe that such research pursuits would contribute unique and meaningful information to our current understanding of the role of movement sounds in motor learning and performance, thus enabling advancement of evidence-based practical recommendations for optimal integration of movement sounds in imagery and modeling interventions.

\section{CONFLICT OF INTEREST}

The authors confirm that this article content has no conflict of interest.

\section{ACKNOWLEDGEMENTS}

Declared none.

\section{REFERENCES}

[1] Cumming J, Clark SE, Ste-Marie DM, et al. The functions of observational learning questionnaire (FOLQ). Psychol Sport Exerc 2005; 6: 517-37.

[2] Hall CR, Mack D, Paivio A, et al. Imagery use by athletes: Development of the Sport Imagery Questionnaire. Int J Sport Psychol 1998; 29: 73-89.

[3] Ste-Marie DM, Law B, Rymal AM, et al. Observation interventions for motor skill learning and performance: An applied model for the use of observation. Int Rev Sport Exerc Psychol 2012; 5: 145-76.

[4] Effenberg AO. Movement sonification: Effects on perception and action. IEEE Multimedia 2005; 12: 53-9.

[5] Lai Q, Shea C, Bruechert L, et al. Auditory modeling enhances relative-timing learning. J Mot Behav 2002; 34(3): 299-307.

[6] Murgia M, Sors F, Vono R, et al. Using auditory stimulation to enhance athletes' strength: An experimental study in weightlifting. Rev Psychol 2012; 19: 13-6.

[7] Schhmitz G, Mohammadi B, Hammer A, et al. Observation of sonified movements engages a basal ganglia frontocortical network. BMC Neurosci 2013; 14: 32.

[8] Munroe-Chandler KJ, Hall CR. Psychological interventions in sport. In: Crocker P, Ed. Introduction to sport psychology: A Canadian perspective. Toronto, ON: Pearson 2007; pp. 184-213.

[9] Silva JM, Stevens DE. Psychological foundations of sport. Boston, MA: Allyn and Bacon 2002.

[10] Morris T, Spittle M, Watt AP. Imagery in sport. Champaign, IL: Human Kinetics 2005.

[11] Blair A, Hall CR, Leyshon G. Imagery effects on the performance of skilled and novice soccer players. J Sports Sci 1993; 11: 95-101.

[12] Callery P, Morris T. The effects of mental practice on the performance of an Australian Rules football skill. In: Serpa S, Alves J, Ferreira V, Paula-Brito A, Eds. Proceedings of VII World Congress of Sport Psychology. Lisbon: ISSP 1993; pp. 646-651.

[13] Millard M, Mahoney C, Wardop J. A preliminary study of mental and physical practice on the kayak wet exit skill. Percept Mot Skills 2001; 92: 977-84.

[14] Hale BD, Whitehouse A. The effects of imagery-manipulated appraisal and intensity and direction of competitive anxiety. Sport Psychol 1998; 12: 40-51.

[15] Martin K, Hall C. Using mental imagery to enhance intrinsic motivation. J Sport Exerc Psychol 1995; 17: 54-69.

[16] Feltz DL, Riessinger CA. Effects of in vivo emotive imagery and performance feedback on self-efficacy and muscular endurance. J Sport Exerc Psychol 1990; 12: 132-43.

[17] Callow N, Hardy L. Types of imagery associated with sport confidence in netball players of varying skill levels. J Appl Sport Psychol 2001; 13: 1-17.

[18] Hall CR. Imagery in sport and exercise. In: Murphy SM, Ed. Handbook of Sport Psychology. New York: Oxford University Press 2001; pp. 529-49. 
[19] Lang PJ. A bio-informational theory of emotional imagery. Psychophysiology 1979; 16(6): 495-512.

[20] Lang PJ. Cognition in emotion: Concept and action. In: Izard C, Kagan J, Zajonc R, Eds. Emotions, cognition and behavior. New York: Cambridge University Press 1985; pp. 192-225.

[21] Paivio A. Cognitive and motivational functions of imagery in human performance. Can J Appl Sport Sci 1985; 10: 22s-28s.

[22] Burhans R, Richman CL, Bergey D. Mental imagery training: Effects on running speed performance. Int J Sport Psychol 1988; 19: 26-32.

[23] Moritz SE, Hall CR, Martin KA, et al. What are confident athletes imaging? An examination of image content. Sport Psychol 1996: 10: 171-9.

[24] Holmes PS, Collins DJ. The PETTLEP approach to motor imagery: A functional equivalence model for sport psychologists. J Appl Sport Psychol 2001; 13: 60-83.

[25] Martin KA, Moritz SE, Hall CR. Imagery use in sport: a literature review and applied model. Sport Psychol 1999; 13: 245-68.

[26] Mahoney MJ, Avener M. Psychology of the elite athlete: an exploratory study. Cogn Ther Res 1977; 1(2): 135-41.

[27] Munroe KJ, Giacobbi PR, Hall C, et al. The four Ws of imagery use: Where, when, why, and what. Sport Psychol 2000; 14: 119-37.

[28] Jenny O, Hall C. A quantitative analysis of athletes' voluntary use of slow motion, real time, and fast motion images. J Appl Sport Psychol 2009; 21(1): 15-30.

[29] Kosslyn SM, Seger C, Pani JR, et al. When is imagery used in everyday life? A diary study. J Ment Imagery 1990; 14(3-4): 13152.

[30] Bandura A. Social foundations of thought and action: A social cognitive theory. Englewood Cliffs, NJ: Prentice-Hall 1986.

[31] McCullagh P, Weiss MR. Modeling: Considerations for motor skill performance and psychological responses. In: Singer RN, Hausenblas HA, Janelle CM, Eds. Handbook of sport psychology. Toronto, Canada: Wiley \& Sons 2001; pp. 205-38.

[32] Williams AM, Davids K, Williams JG. Observational learning. In: Williams AM, Davids K, Williams JG, Eds. Visual perception and action in sport. New York: Routledge 1999; pp. 338-73.

[33] McCullagh P, Law B, Ste-Marie DM. Modeling and performance. In: Murphy SM, Ed. The Oxford Handbook of Sport and Performance Psychology. New York: Oxford University Press 2012; pp. 250-72.

[34] Halliwell W. Providing sport psychology consulting services in professional hockey. Sport Psychol 1990; 4: 369-77.

[35] Leavitt J, Young J, Connelly D. The effects of videotape highlights on state self-confidence. J Appl Res Athlete Coach 1989; 4: 22532.

[36] Templin DP, Vernacchia RA. The effect of highlight music videotapes upon the game performance of intercollegiate basketball players. Sport Psychol 1995; 9: 41-50.

[37] Clark SE, Ste-Marie DM. Peer mastery versus peer coping models: Model type has differential effects on psychological and physical performance measures. J Hum Mov Stud 2002; 43: 179-96.

[38] Clark SE, Ste-Marie DM. The impact of self-as-a-model interventions on children's self-regulation of learning and swimming performance. J Sports Sci 2007; 25(5): 577-86.

[39] Legrain P, d'Arripe-Longueville F, Gernigon C. The influence of trained peer tutoring on tutors' motivation and performance in a French boxing setting. J Sports Sci 2003; 21: 539-50.

[40] Starek J, McCullagh P. The effect of self-modeling on the performance of beginning swimmers. Sport Psychol 1999; 13: 269-87.

[41] Weiss MR, McCullagh P, Smith AL, et al. Observational learning and the fearful child: Influence of peer models on swimming skill performance and psychological responses. Res Q Exerc Sport 1998; 69(4): 380-94.

[42] Ste-Marie DM, Vertes KA, Law B, et al. Learner-controlled selfobservation is advantageous for motor skill acquisition. Front Psychol 2013; 3: 556.

[43] McAuley E. Modeling and self-efficacy:a test of Bandura's model. J Sport Psychol 1985; 7: 283-95.

[44] Feltz DL, Landers DM. The effects of mental practice on motor skill learning and performance: a meta-analysis. J Sport Psychol 1983; 5: 25-57.

[45] Cumming J, Clark SE, Ste-Marie DM, et al. The functions of observational learning questionnaire (FOLQ). Psychol Sport Exerc 2005; 6(5): 517-37.
[46] Law B, Hall C. Observational learning use and self-efficacy beliefs in adult sport novices. Psychol Sport Exerc 2009; 10: 263-70.

[47] Maddison R, Prapavessis H, Clatworthy M. Modeling and rehabilitation following anterior cruciate ligament reconstruction. Ann Behav Med 2006; 31(1): 89-98

[48] Ste-Marie DM, Law B, Rymal AM, et al. Observation interventions for motor skill learning and performance: an applied model for the use of observation. Int Rev Sport Exerc Psychol 2012; 5(2): 145-76.

[49] Fournier JF, Deremaux S, Bernier M. Content, characteristics and function of mental images. Psychol Sport Exerc 2008; 9: 734-48.

[50] Jenny O, Munroe-Chandler KJ. The effects of image speed on the performance of a soccer task. Sport Psychol 2008; 22(1): 1-17.

[51] Weinberg RS, Gould D. Foundations of sport and exercise psychology. $3^{\text {rd }}$ ed. Champaign, IL: Human Kinetics 2003.

[52] Guillot A, Collet C. Construction of the motor imagery integrative model in sport: a review and theoretical investigation of motor imagery use. Int Rev Sport Exerc Psychol 2008; 1(1): 31-44.

[53] Jenny O, Hall C. Does speed matter? a qualitative analysis of the 'why' of athletes' voluntary image speed use. J Imagery Res Sport 2013; 8(1): 1-12

[54] Weinberg RS, Gould D. Foundations of sport and exercise psychology, $5^{\text {th }}$ ed. Champaign, IL: Human Kinetics 2011.

[55] George TR, Feltz DL, Chase MA. Effects of model similarity on self-efficacy and muscular endurance: a second look. J Sport Exerc Psychol 1992; 14(3): 237-48.

[56] Lirgg CD, Feltz DL. Teacher versus peer models revisited: effects on motor performance and self-efficacy. Res Q Exerc Sport 1991; 62(2): 217-24.

[57] McCullagh P, Meyer KN. Learning versus correct models: Influence of model type on the learning of a free-weight squat lift. Res Q Exerc Sport 1997; 68(1): 56-61

[58] Weir PL, Leavitt JL. Effects of model's skill level and model's knowledge of results on the performance of a dart throwing task. Hum Mov Sci 1990; 9(3): 369-83.

[59] Lai Q, Shea CH, Little M. Effects of modeled auditory information on a sequential timing task. Res Q Exerc Sport 2000; 71(4): 34956.

[60] Shea CH, Wulf G, Park JH, et al. Effects of an auditory model on the learning of relative and absolute timing. J Mot Behav 2001; 33(2): 127-38.

[61] Agostini T, Righi G, Galmonte A, et al. The relevance of auditory information in optimizing hammer throwers performance. In Pascolo PB, Ed. Biomechanics and sports. Vienna, Austria: Springer 2004; pp. 67-74.

[62] Gould D, Weiss M. The effects of model similarity and model talk on self-efficacy and muscular endurance. J Sport Psychol 1981; 3(1): 17-29.

[63] McCullagh P. Model similarity effect on motor performance. J Sport Psychol 1987; 9: 249-60.

[64] Murgia M, Hohmann T, Galmonte A, et al. Recognizing one's own motor actions through sound: the role of temporal factors. Perception 2012; 41: 976-87.

[65] Buccino G, Binkofski F, Fink GR, et al. Action observation activates premotor and parietal areas in a somatotopic manner: An fMRI study. Eur J Neurosci 2001; 13: 400-4.

[66] Ehrsson HH, Geyer S, Naito E. Imagery of voluntary movement of fingers, toes, and tongue activates corresponding body-part-specific motor representations. J Neurophysiol 2003; 90: 3304-16.

[67] Halpern AR, Zatorre RJ. When that tune runs through your head: a PET investigation of auditory imagery for familiar melodies. Cerebral Cortex 1999; 9(7): 697-704.

[68] Woods EA, Hernandez AE, Wagner VE, et al. Expert athletes activate somatosensory and motor planning regions of the brain when passively listening to familiar sports sounds. Brain Cogn 2014; 87: 122-33.

[69] Cesari P, Camponogara I, Papetti S, et al. Might as well jump: sound affects muscle activation in skateboarding. PLoS One 2014; 9(3): e90156.

[70] Kennel C, Pizzera A, Hohmann T, et al. The perception of natural and modulated movement sounds. Perception 2014; 43(8): 796804.

[71] Murgia M, Santoro I, Tamburini G, et al. Ecological sounds affect breath duration more than artificial sounds. Psychol Res 2015; [epub ahead of print]. DOI 10.1007/s00426-015-0647-z 
[72] Dowrick PW. A Review of self-modeling and related interventions. Appl Prev Psychol 1999; 8: 23-9.

[73] McCullagh P. Model status as a determinant of attention in observational learning and performance. J Sport Psychol 1986; 8: 31931 .

[74] Hebert EP, Landin D. Effects of a learning model and augmented feedback on tennis skill acquisition. Res Q Exerc Sport 1994; 65(3): 250-7.

[75] Rymal AM, Martini R, Ste-Marie DM. Self-regulatory processes employed during self-modeling: a qualitative analysis. Sport Psychol 2010; 24: 1-15.

[76] Di Pellegrino G, Fadiga L, Fogassi L, et al. Understanding motor events: a neurophysiological study. Exp Brain Res 1992; 91(1): 176-80.

[77] Halpern AR, Zatorre RJ, Bouffard M, et al. Behavioral and neural correlates of perceived and imagined musical timbre. Neuropsychologia 2004; 42(9): 1281-92.

[78] Zatorre R, Halpern A, Perry D, et al. Hearing in the mind's ear: a PET investigation of musical imagery and perception. J Cogn Neurosci 1996; 8(1): 29-46.

[79] Keller PE, Appel M. Individual differences, auditory imagery, and the coordination of body movements and sounds in musical ensembles. Music Percept 2010; 28(1): 27-46.
[80] Hubbard TL. Auditory imagery contains more than audition. In: Lacey S, Lawson R, Eds. Multisensory Imagery. New York: Springer 2013; pp. 221-47.

[81] Intons-Peterson MJ. The role of loudness in auditory imagery. Mem Cogn 1980; 8: 385-93.

[82] Hall CR, Martin KA. Measuring movement imagery abilities: A revision of the movement imagery questionnaire. J Mental Imagery 1997; 21: 143-54.

[83] Roberts R, Callow N, Hardy L, et al. Movement imagery ability: Development and assessment of a revised version of the vividness of movement imagery questionnaire. J Sport Exerc Psychol 2008; 30: 200-21.

[84] Highben Z, Palmer C. Effects of auditory and motor mental practice in memorized piano performance. Bull Counc Res Music Educ 2004; 159: 58-65.

[85] Keller PE, Dalla Bella S, Koch I. Auditory imagery shapes movement timing and kinematics: Evidence from a musical task. J Exp Psychol Hum 2010; 36(2): 508.

[86] Kennel C, Streese L, Pizzera A, et al. Auditory reafferences: The influence of real-time feedback on movement control. Front Psychol 2015; 6: 69 .

(C) Jenny $\mathrm{O}$ et al.; Licensee Bentham Open.

This is an open access articles licensed under the terms of the Creative Commons Attribution-Non-Commercial 4.0 International Public License (CC BY-NC 4.0) (https://creativecommons.org/licenses/by-nc/4.0/legalcode), which permits unrestricted, non-commercial use, distribution and reproduction in any medium, provided that the work is properly cited. 